Article

\title{
Synthesis and Bioactivity Evaluation of
} $N$-Arylsulfonylindole Analogs Bearing a Rhodanine Moiety as Antibacterial Agents

\author{
Ming-Xia Song, Song-Hui Li, Jiao-Yang Peng, Ting-Ting Guo, Wen-Hui Xu, Shao-Feng Xiong and \\ Xian-Qing Deng *
}

Basic Medical and Pharmacy College, Jinggangshan University, Ji'an 343009, Jiangxi, China; freexiaoxiao83@aliyun.com (M.-X.S.); 18379698767@163.com (S.-H.L.); sakura367468@163.com (J.-Y.P.); m18379680319@163.com (T.-T.G.); 13687965920@163.com (W.-H.X.); 18370669382@163.com (S.-F.X.)

* Correspondence: xqdeng@jgsu.edu.cn; Tel.: +86-157-7919-7280

Received: 28 April 2017; Accepted: 9 June 2017; Published: 14 June 2017

\begin{abstract}
Due to the rapidly growing bacterial resistance to antibiotics and the scarcity of novel agents under development, bacterial infections are still a pressing global problem, making new types of antibacterial agents, which are effective both alone and in combination with traditional antibiotics, urgently needed. In this paper, seven series of $N$-arylsulfonylindole analogs 5-11 bearing rhodanine moieties were synthesized, characterized, and evaluated for antibacterial activity. According to the in vitro antimicrobial results, half of the synthesized compounds showed potent inhibition against four Gram-positive bacteria, with MIC values in the range of $0.5-8 \mu \mathrm{g} / \mathrm{mL}$. For multidrug-resistant strains, compounds $6 \mathbf{a}$ and $6 \mathrm{c}$ were the most potent, with MIC values of $0.5 \mu \mathrm{g} / \mathrm{mL}$, having comparable activity to gatifloxacin, moxiflocaxin and norfloxacin and being 128 -fold more potent than oxacillin (MIC $=64 \mu \mathrm{g} / \mathrm{mL}$ ) and 64 -fold more active than penicillin (MIC $=32 \mu \mathrm{g} / \mathrm{mL}$ ) against Staphylococcus aureus ATCC 43300.
\end{abstract}

Keywords: rhodanine; $N$-arylsulfonylindole; propanoic acid; antibacterial activity

\section{Introduction}

Bacterial drug resistance has emerged and become increasingly serious on a global scale, both in developed and developing countries [1]. Drug-resistant bacteria, such as methicillin-resistant Staphylococcus aureus (MRSA), multi-drug resistant Escherichia coli, and multi-drug resistant Pseudomonas aeruginosa, cause lethal diseases and cause great difficulties in the treatment of nosocomial infections [2-5], which severely threaten global public health while resulting in very large economic costs [6]. Currently the development of new antimicrobial drugs cannot keep pace with the development of bacterial drug resistance, and the number of new antibiotics approved for marketing per year is declining continuously. Taking advantage of pathogens virulence is an alternative strategy to inhibit antibiotic resistance development, and some studies have focused on anti-virulence agents against Gram-positive pathogens and Gram-negative pathogens [7,8]. However, this strategy has some weaknesses that limiting its development, such as the lack of a good way to assessing the efficacy and the inapplicability in immunocompromised patients. Over the last decade, a few new antibiotics (e.g., linezolid, ceftolozane, telavancin, ceftaroline fosamil, Xifaxan ${ }^{\circledR}$, and daptomycin) were approved and dozens of antibiotics are currently in Phase 2 or Phase 3 clinical trials [9]. However, these drugs cannot address the entire spectrum of bacteria resistance [10], therefore, there is an urgent need to develop new antimicrobial agents, especially those with a new drug target or with the ability to overcome drug resistance. 
Indole, an intercellular signaling molecule, regulates various aspects of bacterial physiology, including spore formation, plasmid stability, resistance to drugs, biofilm formation, and virulence. The amino acid tryptophan is an indole derivative and the precursor of the neurotransmitter serotonin. Up to now, indoles have displayed important physiological functions and potent pharmacological activities, including anti-inflammatory and antioxidant [11], antineoplastic [12-14], antimicrobial [15,16], antiviral $[14,17,18]$, and anti-HIV activity $[19,20]$. $N$-Arylsulfonylindoles, as a kind of indole derivative, have received a great deal of attention in the field of chemical drug research, behaving as 5- $\mathrm{HT}_{6}$ receptor antagonists [21], anti-AIDS drugs [22], and antifungal agents [23]. However, the antibacterial activity of $\mathrm{N}$-arylsulfonylindoles has not yet been reported to our knowledge.

Previously, we reported a series of rhodanine derivatives (compounds I, Scheme 1) which showed good inhibitory activity against Gram-positive bacteria (including multidrug-resistant clinical isolates) [24-29], which suggested that the rhodanine moiety was an important fragment for antimicrobial activity. Fragment-based drug discovery and skeleton migration strategy is a rational technique for molecular modification and drug design when some active fragments are obtained. Based upon these observations, and as part of our ongoing program aiming at the discovery and development of bioactive molecules, in this work, seven series of $N$-arylsulfonyl-3-substituted indoles 5-11 (Scheme 1) were designed using compound I as the lead compound. The target compounds were prepared by combining the rhodanine groups with $\mathrm{N}$-arylsulfonylindoles, and their anti-bacterial activities were screened.
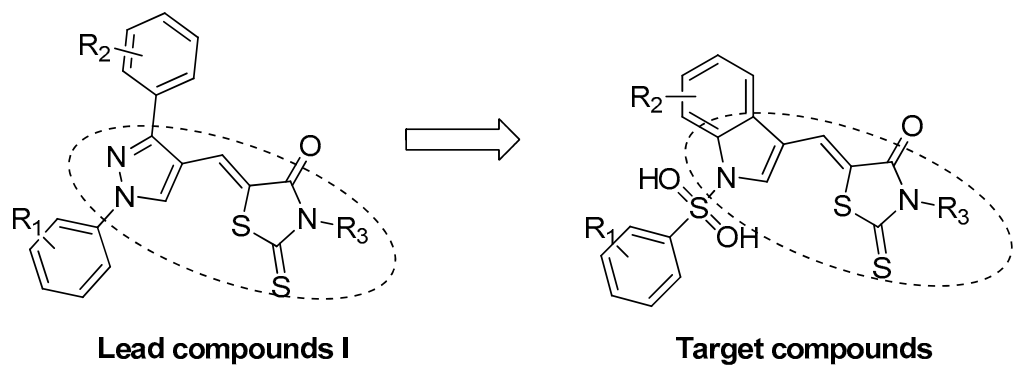

Scheme 1. The design of the target compounds 5-11.

Chen et al. [30] have reported the synthesis and antibacterial activity evaluation of chalcone derivatives containing a rhodanine-3-acetic acid moiety, and found that the antibacterial activity of compounds with halogen and methyl substituents on the phenyl group was obviously higher than that of compounds with other substituents. In view of these findings, in this paper, only halogen and methyl substituents were chosen on the indole and phenyl sulfonyl moieties.

\section{Results and Discussion}

\subsection{Chemistry}

The target compounds were synthesized as outlined in Scheme 2. A series of 3-substituted-5((1-(phenylsulfonyl)-1H-indol-3-yl)methylene)-2-thioxothiazolidin-4-ones were synthesized using $1 H$-indole-3-carbaldehydes as the starting material. Firstly, benzenesulfonyl chlorides $\mathbf{1 a}, \mathbf{1} \mathbf{b}$ were reacted with $1 \mathrm{H}$-indole-3-carbaldehydes $\mathbf{2 a - 2} \mathbf{c}$ in the presence of anhydrous potassium carbonate $\left(\mathrm{K}_{2} \mathrm{CO}_{3}\right)$ at $40{ }^{\circ} \mathrm{C}$ to give 1-(phenylsulfonyl)- $1 \mathrm{H}$-indole-3-carbaldehydes $3 \mathbf{a}-\mathbf{f}$, which were directly used in the next step without purification. Next compounds $3 \mathbf{a}-\mathbf{f}$ were subjected to a Knoevenagel condensation reaction with appropriate $N$-substituted rhodanines to provide seven new series of target compounds 5-11. The structures of the products were well characterized by ${ }^{1} \mathrm{H}-\mathrm{NMR},{ }^{13} \mathrm{C}-\mathrm{NMR}$, and high-resolution mass spectrometry. 


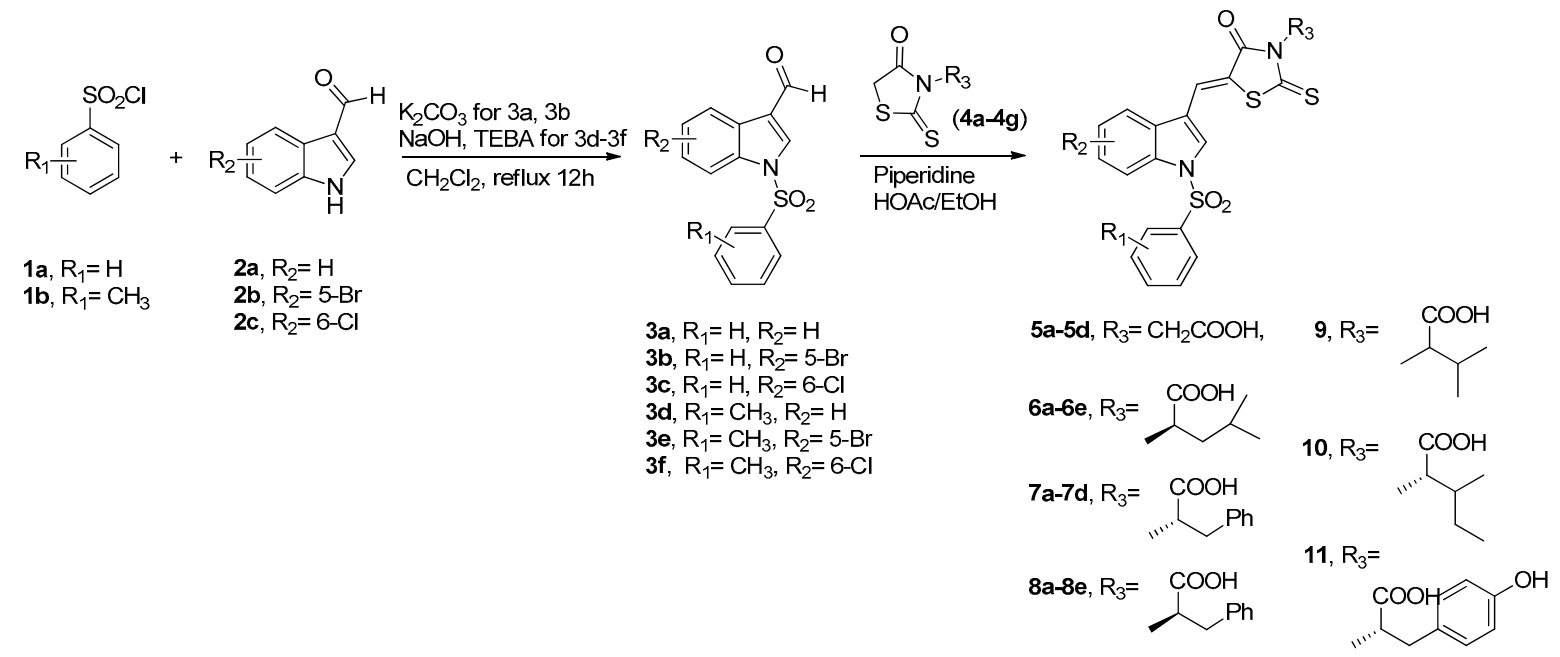

Scheme 2. The synthesis route of compounds 5-11.

\subsection{Antimicrobial Activity}

All of the target compounds 5-11 were evaluated for their in vitro anti-bacterial activity using a serial dilution method to obtain the minimum inhibitory concentration (MIC) against five Gram-positive strains (S. aureus (CMCC(B) 26003 and CMCC 25923, Streptococcus pyogenes CMCC 32067, Enterococcus faecalis CMCC 29212, and Bacillus subtilis CMCC 63501), four Gram-negative strains (E. coli (CMCC 25922 and CMCC 44568) and P. aeruginosa (CMCC 27853 and CMCC 10104))m as well as two methicillin-resistant clinical isolates (S. aureus ATCC 43300 and ATCC 33591). Gatifloxacin, moxifloxacin, norfloxacin, oxacillin, and penicillin were used as positive control drugs.

Preliminarily, compounds 5-11 were screened for their activity against five Gram-positive strains and four Gram-negative strains. Initial screening results described as MIC values are presented in Table 1. The results illustrate that the inhibition of the seven series of derivatives against Gram-positive strains (effective against four bacteria) is in general superior to that of Gram-negative strains (effective against one bacterium). For Gram-positive strains, more than half of the tested compounds showed potent inhibition activity against S. aureus (CMCC (B) 26003 and CMCC 25923), with MIC values in the range of $0.5-4 \mu \mathrm{g} / \mathrm{mL}$. Half of the target compounds exhibited moderate activity against $E$. faecalis

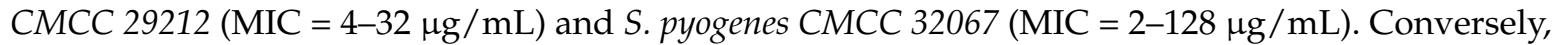
five positive control agents did not exhibit inhibition activity for the two strains (MICs $>128 \mu \mathrm{g} / \mathrm{mL}$ ). All compounds, however, had no effect on B. subtilis CMCC 63501 at $128 \mu \mathrm{g} / \mathrm{mL}$. For Gram-negative strains, only a few of compounds showed moderate activity against P. aeruginosa CMCC 10104 with MICs of 4-64 $\mu \mathrm{g} / \mathrm{mL}$, while showing no inhibitory activity against three other strains at $128 \mu \mathrm{g} / \mathrm{mL}$. The study found that, among the compounds synthesized, compound $\mathbf{8 b}$ was the most active compound against two $S$. aureus with MIC values of 1 or $0.5 \mu \mathrm{g} / \mathrm{mL}$, along with MIC $=2 \mathrm{or} 4 \mu \mathrm{g} / \mathrm{mL}$ against S. pyogenes CMCC 32067 and E. faecalis CMCC 29212, respectively.

In the following trials, five compounds $(\mathbf{6 a}, \mathbf{6 c}, \mathbf{8 a - c})$ were chosen to evaluate their inhibitory activity against two clinical isolates of multidrug-resistant Gram-positive bacterial strains (S. aureus ATCC 43300 and S. aureus ATCC 33591), whose MICs against S. aureus (CMCC(B) 26003 or CMCC 25923 are less than $1 \mu \mathrm{g} / \mathrm{mL}$. The results are listed in Table 2 . The data illustrated that five compounds had excellent inhibitory activities against the two multidrug-resistant strains, with MICs of $0.5,1$, or $2 \mu \mathrm{g} / \mathrm{mL}$. Among them, compounds $6 \mathbf{a}$ and $\mathbf{6 c}$ were the most potent, with MIC values of $0.5 \mu \mathrm{g} / \mathrm{mL}$, having comparable activity to gatifloxacin, moxiflocaxin and norfloxacin, while being 128 -fold more potent than oxacillin (MIC $=64 \mu \mathrm{g} / \mathrm{mL}$ ) and 64-fold more active than penicillin (MIC $=32 \mu \mathrm{g} / \mathrm{mL}$ ) against $S$. aureus ATCC 43300. 
Table 1. Inhibitory activity (MIC, $\mu \mathrm{g} / \mathrm{mL}$ ) of compounds 5-11 against Gram-positive and Gram-negative bacteria.

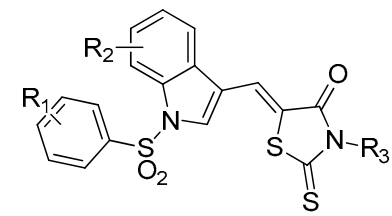

\begin{tabular}{|c|c|c|c|c|c|c|c|c|c|c|c|c|}
\hline \multirow{2}{*}{ Compd. } & \multirow{2}{*}{$\mathbf{R}_{\mathbf{1}^{-}}$} & \multirow{2}{*}{$\mathbf{R}_{2-}$} & \multirow{2}{*}{$\mathbf{R}_{3}-$} & \multicolumn{5}{|c|}{ Gram-Positive Strains } & \multicolumn{4}{|c|}{ Gram-Negative Strains } \\
\hline & & & & $26003^{a}$ & $25923^{b}$ & $32067^{c}$ & $29212^{d}$ & $635011^{e}$ & $25922^{f}$ & $44568^{\mathrm{g}}$ & $27853^{h}$ & $10104^{i}$ \\
\hline $5 a$ & $\mathrm{H}$ & $5-\mathrm{Br}$ & $\mathrm{CH}_{2} \mathrm{COOH}$ & 8 & 8 & $>128$ & 32 & $>128$ & $>128$ & $>128$ & $>128$ & 32 \\
\hline $5 b$ & $\mathrm{H}$ & $6-\mathrm{Cl}$ & $\mathrm{CH}_{2} \mathrm{COOH}$ & $>128$ & $>128$ & $>128$ & $>128$ & $>128$ & $>128$ & $>128$ & $>128$ & $>128$ \\
\hline $5 c$ & $\mathrm{CH}_{3}$ & $5-\mathrm{Br}$ & $\mathrm{CH}_{2} \mathrm{COOH}$ & 8 & 16 & $>128$ & $>128$ & $>128$ & $>128$ & $>128$ & $>128$ & $>128$ \\
\hline $5 d$ & $\mathrm{CH}_{3}$ & $6-\mathrm{Cl}$ & $\mathrm{CH}_{2} \mathrm{COOH}$ & 4 & 4 & $>128$ & 32 & $>128$ & $>128$ & $>128$ & $>128$ & 32 \\
\hline $6 a$ & $\mathrm{H}$ & $\mathrm{H}$ & & 2 & 1 & 16 & 8 & $>128$ & $>128$ & $>128$ & $>128$ & 8 \\
\hline $6 b$ & $\mathrm{H}$ & $6-\mathrm{Cl}$ & & 4 & 2 & $>128$ & 16 & $>128$ & $>128$ & $>128$ & $>128$ & 16 \\
\hline $6 c$ & $\mathrm{CH}_{3}$ & $\mathrm{H}$ & & 2 & 1 & $>128$ & 4 & $>128$ & $>128$ & $>128$ & $>128$ & 4 \\
\hline $6 d$ & $\mathrm{CH}_{3}$ & $5-\mathrm{Br}$ & & 2 & 2 & $>128$ & 4 & $>128$ & $>128$ & $>128$ & $>128$ & 4 \\
\hline $6 e$ & $\mathrm{CH}_{3}$ & $6-\mathrm{Cl}$ & & 64 & $>128$ & $>128$ & $>128$ & $>128$ & $>128$ & $>128$ & $>128$ & $>128$ \\
\hline $7 a$ & $\mathrm{H}$ & $\mathrm{H}$ & & 2 & 2 & 128 & 4 & $>128$ & $>128$ & $>128$ & $>128$ & 4 \\
\hline $7 b$ & $\mathrm{H}$ & $6-\mathrm{Cl}$ & & 32 & 16 & $>128$ & 8 & $>128$ & $>128$ & $>128$ & $>128$ & 8 \\
\hline $7 c$ & $\mathrm{CH}_{3}$ & $\mathrm{H}$ & & 2 & 16 & $>128$ & $>128$ & $>128$ & $>128$ & $>128$ & $>128$ & $>128$ \\
\hline $7 \mathrm{~d}$ & $\mathrm{CH}_{3}$ & $5-\mathrm{Br}$ & & 2 & 2 & $>128$ & 16 & $>128$ & $>128$ & $>128$ & $>128$ & 16 \\
\hline
\end{tabular}


Table 1. Cont.

\begin{tabular}{|c|c|c|c|c|c|c|c|c|c|c|c|c|}
\hline \multirow{2}{*}{ Compd. } & \multirow{2}{*}{$\mathbf{R}_{\mathbf{1}^{-}}$} & \multirow{2}{*}{$\mathbf{R}_{2-}$} & \multirow{2}{*}{$\mathbf{R}_{3}-$} & \multicolumn{5}{|c|}{ Gram-Positive Strains } & \multicolumn{4}{|c|}{ Gram-Negative Strains } \\
\hline & & & & $26003^{a}$ & $25923^{b}$ & $32067^{c}$ & $29212^{d}$ & $63501^{e}$ & $25922^{f}$ & $44568 \mathrm{~g}$ & $27853^{h}$ & 10104 \\
\hline $8 a$ & $\mathrm{H}$ & $\mathrm{H}$ & & 2 & 1 & 4 & 16 & $>128$ & $>128$ & $>128$ & $>128$ & 16 \\
\hline $8 b$ & $\mathrm{H}$ & 6-Cl & & 1 & 0.5 & 2 & 4 & $>128$ & $>128$ & $>128$ & $>128$ & 4 \\
\hline $8 c$ & $\mathrm{CH}_{3}$ & $\mathrm{H}$ & & 1 & 1 & 128 & $>128$ & $>128$ & $>128$ & $>128$ & $>128$ & $>128$ \\
\hline $8 d$ & $\mathrm{CH}_{3}$ & $5-\mathrm{Br}$ & & 2 & 2 & $>128$ & 64 & $>128$ & $>128$ & $>128$ & $>128$ & 64 \\
\hline $8 e$ & $\mathrm{CH}_{3}$ & 6-Cl & & 2 & 2 & $>128$ & 64 & $>128$ & $>128$ & $>128$ & $>128$ & 64 \\
\hline 9 & $\mathrm{H}$ & $\mathrm{H}$ & & 4 & 4 & $>128$ & 32 & $>128$ & $>128$ & $>128$ & $>128$ & 32 \\
\hline 10 & $\mathrm{H}$ & $\mathrm{H}$ & & 2 & 2 & 16 & 16 & $>128$ & $>128$ & $>128$ & $>128$ & 16 \\
\hline 11 & $\mathrm{H}$ & $\mathrm{H}$ & & $>128$ & 8 & $>128$ & 32 & $>128$ & $>128$ & $>128$ & $>128$ & 32 \\
\hline Gatifloxacin & - & - & - & 0.125 & 0.125 & $>128$ & $>128$ & 1 & 2 & 0.125 & 0.125 & 2 \\
\hline Moxifloxacin & - & - & - & 0.125 & 0.125 & $>128$ & $>128$ & 1 & 2 & 0.125 & 0.125 & 2 \\
\hline Norfloxacin & - & - & - & 0.125 & 0.125 & $>128$ & $>128$ & 1 & 2 & 0.125 & 0.125 & 2 \\
\hline Oxacillin & - & - & - & 0.125 & 0.125 & $>128$ & $>128$ & 128 & $>128$ & 128 & $>128$ & $>128$ \\
\hline penicillin & - & - & - & 0.125 & 0.125 & $>128$ & $>128$ & 128 & 128 & 128 & $>128$ & $>128$ \\
\hline
\end{tabular}

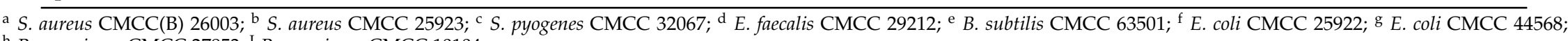
h P. aeruginosa CMCC 27853; ${ }^{\text {I }}$ P. aeruginosa CMCC 10104. 
Table 2. Inhibitory activity (MIC, $\mu \mathrm{g} / \mathrm{mL}$ ) of compounds $\mathbf{6 a}, \mathbf{6 c}$ and $\mathbf{8 a}-\mathbf{c}$ against clinical isolates of multidrug-resistant strains.

\begin{tabular}{|c|c|c|c|c|c|}
\hline \multirow[t]{2}{*}{ Compound } & \multirow[t]{2}{*}{$\mathbf{R}_{\mathbf{1}^{-}}$} & \multirow[t]{2}{*}{$\mathbf{R}_{2-}$} & \multirow[t]{2}{*}{$\mathbf{R}_{3^{-}}$} & \multicolumn{2}{|c|}{$\begin{array}{l}\text { Multidrug-Resistant } \\
\text { Gram-Positive Strains }\end{array}$} \\
\hline & & & & $43300^{a}$ & $33591^{b}$ \\
\hline $6 a$ & $\mathrm{H}$ & $\mathrm{H}$ & & 0.5 & 1 \\
\hline $6 c$ & $\mathrm{CH}_{3}$ & $\mathrm{H}$ & & 0.5 & 1 \\
\hline $8 a$ & $\mathrm{H}$ & $\mathrm{H}$ & & 1 & 2 \\
\hline $8 b$ & $\mathrm{H}$ & $6-\mathrm{Cl}$ & & 2 & 2 \\
\hline $8 c$ & $\mathrm{CH}_{3}$ & $\mathrm{H}$ & & 1 & 2 \\
\hline Gatifloxacin & - & - & - & 0.5 & 0.25 \\
\hline Moxifloxacin & - & - & - & 0.5 & 0.25 \\
\hline Norfloxacin & - & - & 一 & 0.5 & 0.25 \\
\hline Oxacillin & - & - & - & 64 & 8 \\
\hline Penicillin & - & - & - & 32 & $>32$ \\
\hline
\end{tabular}

a S. aureus ATCC $43300 ;{ }^{\text {b }}$ S. aureus ATCC 33591.

\subsection{Cytotoxicity}

The cytotoxic properties of compounds $\mathbf{6 a}, \mathbf{8 b}$, and $8 \mathbf{c}$ were also investigated on HEK 293T cells using the CCK-8 method and the results are shown in Table 3. Compounds $\mathbf{6 a}, \mathbf{8 b}$, and $\mathbf{8 c}$, with $\mathrm{IC}_{50}$ values of $36.90,54.09$, and $32.28 \mu \mathrm{g} / \mathrm{mL}$, respectively, were not cytotoxic at concentrations in the range of $0.5-16 \mu \mathrm{g} / \mathrm{mL}$. The comparison between the MIC and $\mathrm{IC}_{50}$ values of the tested compounds suggests that compounds $\mathbf{6 a}, \mathbf{8 b}$, and $\mathbf{8 c}$ exhibit in vitro antibacterial activity at non-cytotoxic concentrations.

Table 3. Cytotoxic activity of compounds $\mathbf{6 a}, \mathbf{8 b}$ and $\mathbf{8 c}$ on the HEK 293 T cells.

\begin{tabular}{cc}
\hline Compound & IC $_{\mathbf{5 0}}(\mu \mathrm{g} / \mathrm{mL})^{\mathbf{a}}$ \\
\hline $\mathbf{6 a}$ & 36.90 \\
$\mathbf{8 b}$ & 54.09 \\
$\mathbf{8 c}$ & 32.28 \\
\hline the concentration required to inhibit $50 \%$ of cell growth.
\end{tabular}

\subsection{The Structure-Activity Relationships (SARs) Analysis}

Based on the present data of the synthesized compounds, simple SARs could be proposed. For the derivatives bearing weak electron-donating substituents $\left(\mathrm{R}_{1}=-\mathrm{CH}_{3}\right)$, it seems that there is no obvious impact on the antibacterial activity in comparison with non-substituted compounds $\left(R_{1}=H\right)$. In series 6, for example, the MIC values against CMCC 26003 of compound $\mathbf{6 c}\left(\mathrm{R}_{1}=-\mathrm{CH}_{3}\right)$ and compound $\mathbf{6 a}$ $\left(\mathrm{R}_{1}=\mathrm{H}\right)$ were both $2 \mu \mathrm{g} / \mathrm{mL}$, and the same result was discovered in the series 7 . As evidenced from Table 1 , this could lead to the conclusion that a general inhibitory activity order of seven series of target compounds was series $6,8>$ series $7,10>$ series $9>$ series 5,11 , but the differences are not remarkable. Upon comparison of series $\mathbf{7}$ and $\mathbf{8}$, it can be found that the activity of the $R$-configuration compounds appears to be slightly better than that of $S$-configuration compounds. 


\section{Materials and Methods}

\subsection{Instruments and Reagents}

All of the reagents and solvents were purchased from Aladdin (Shanghai, China) or Sinopharm Chemical Reagent Co. Ltd. (Shanghai, China), and were used as received. Melting points were determined in open capillary tubes and are uncorrected. Reaction courses were monitored by thin-layer chromatography on silica gel-precoated F254 plates (Merck, Darmstadt, Germany). Developed plates were examined with UV lamps $(254 \mathrm{~nm})$. Nuclear magnetic resonance spectroscopy was performed on an AV-300 spectrometer (Bruker, Zurich, Switzerland) operating at $300 \mathrm{MHz}$ for ${ }^{1} \mathrm{H}$ and $75 \mathrm{MHz}$ for ${ }^{13} \mathrm{C}$ and using DMSO- $d_{6}$ as solvent and tetramethylsilane as the internal standard. Electrospray Ionization Mass Spectrometry (ESI-MS) experiments were performed on an IT-TOF mass spectrometer (Shimadzu, Tokyo, Japan) in negative ion mode. Specific optical rotation was measured on a Digital automatic polariscope JASCO P-1020 (JASCO, Tokyo, Japan).

\subsection{Synthesis Method and Spectral Data}

\subsubsection{General Procedure for the Preparation of Compounds $\mathbf{3 a}-\mathbf{3 b}$}

To a dry dichloromethane solution $(10 \mathrm{~mL})$ of the appropriate $1 H$-indole-3-carbaldehydes ( $2 \mathrm{mmol})$, anhydrous potassium carbonate $(6 \mathrm{mmol})$ and benzenesulfonyl chlorides $(4 \mathrm{mmol})$ in dry dichloromethane $(20 \mathrm{~mL})$ were added, and the mixture was stirred for $12 \mathrm{~h}$ at $40{ }^{\circ} \mathrm{C}$. After the completion of the reaction, excess solvent was removed under reduced pressure to obtain a yellow crude solid of $\mathbf{3 a}-\mathbf{3 b}$ which was directly used in the next step without purification.

\subsubsection{General Procedure for the Preparation of Compounds $\mathbf{3 c}-\mathbf{3 f}$}

To a solution of the appropriate $1 H$-indole-3-carbaldehydes $(1 \mathrm{mmol})$ in dry dichloromethane (30 mL), sodium hydroxide $(1.75 \mathrm{mmol})$, benzyltriethylammonium chloride (TEBA, $0.1 \mathrm{mmol})$, and benzenesulfonyl chlorides $(1.2 \mathrm{mmol})$ were added and stirred for $12 \mathrm{~h}$ at room temperature. After the completion of the reaction, $15 \mathrm{~mL}$ water was added into the mixture. Then the mixture was extracted with dichloromethane $(30 \mathrm{~mL} \times 3)$. The combined organic layers were dried over anhydrous $\mathrm{MgSO}_{4}$ before being concentrated in vacuo. The crude products $\mathbf{3 c}-\mathbf{3 f}$ obtained were directly used in the next step without purification.

\subsubsection{General Procedure for the Preparation of Compounds 5-11}

A mixture of 3 ( $1 \mathrm{mmol})$, corresponding rhodanine $(1 \mathrm{mmol}), 10$ drops glacial acetic acid and 10 drops piperidine in ethanol $(20 \mathrm{~mL})$ was refluxed for $16 \mathrm{~h}$. After cooling, the solvent was evaporated in vacuo, followed by the purification of the resulting residue by silica gel column chromatography (dichloromethane/methanol $=100 / 1$ or $150 / 1$ ) to obtain a yellow solid 5-11.

\subsubsection{Spectral Data}

2-(5-((5-Bromo-1-(phenylsulfonyl)-1H-indol-3-yl)methylene)-4-oxo-2-thioxothiazolidin-3-yl)acetic acid (5a). Yellow solid; yield 45\%; m.p. $242-246{ }^{\circ} \mathrm{C} .{ }^{1} \mathrm{H}-\mathrm{NMR}: \delta 4.64$ (s, 2H, NCH ), 7.65-8.38 (m, 10H, Ar-H, $\mathrm{CH}=\mathrm{C}), 12.80$ (br.s, 1H, COOH). ${ }^{13} \mathrm{C}-\mathrm{NMR}: \delta$ 192.57, 167.35, 166.27, 136.47, 135.98, 133.12, 131.05, $130.66,129.50,129.00,127.79,127.75,125.95,123.79,117.92,116.80,115.59,53.18$. ESI-HRMS calcd. for $\mathrm{C}_{20} \mathrm{H}_{12} \mathrm{BrN}_{2} \mathrm{O}_{5} \mathrm{~S}_{3}^{-}\left([\mathrm{M}-\mathrm{H}]^{-}\right)$: 534.9097; found: 534.9118 .

2-(5-((6-Chloro-1-(phenylsulfonyl)-1H-indol-3-yl)methylene)-4-oxo-2-thioxothiazolidin-3-yl)acetic acid (5b). Yellow solid; yield 47\%; m.p. $206-208{ }^{\circ} \mathrm{C} .{ }^{1} \mathrm{H}-\mathrm{NMR}: \delta 4.40\left(\mathrm{~s}, 2 \mathrm{H}, \mathrm{NCH}_{2}\right), 7.44-8.24(\mathrm{~m}, 10 \mathrm{H}, \mathrm{Ar}-\mathrm{H}$, $\mathrm{CH}=\mathrm{C}), 9.24$ (br.s, 1H, COOH). ${ }^{13} \mathrm{C}-\mathrm{NMR}: \delta$ 192.54, 167.13, 166.62, 136.53, 136.02, 134.67, 131.51, $130.75,128.35,127.99,127.76,125.39,124.67,122.56,121.59,117.33,113.31,43.98$. ESI-HRMS calcd. for $\mathrm{C}_{20} \mathrm{H}_{12} \mathrm{ClN}_{2} \mathrm{O}_{5} \mathrm{~S}_{3}^{-}\left([\mathrm{M}-\mathrm{H}]^{-}\right): 490.9602$; found: 490.9615. 
2-(5-((5-Bromo-1-tosyl-1H-indol-3-yl)methylene)-4-oxo-2-thioxothiazolidin-3-yl)acetic acid (5c). Yellow solid; yield 44\%; m.p. $226{ }^{\circ} \mathrm{C} .{ }^{1} \mathrm{H}-\mathrm{NMR}$ : $\delta 2.33$ (s, 3H, $\left.\mathrm{CH}_{3}\right), 4.40\left(\mathrm{~s}, 2 \mathrm{H}, \mathrm{NCH}_{2}\right), 7.37-8.40(\mathrm{~m}, 9 \mathrm{H}, \mathrm{Ar}-\mathrm{H}$, $\mathrm{CH}=\mathrm{C}), 9.31$ (br.s, $1 \mathrm{H}, \mathrm{COOH}) .{ }^{13} \mathrm{C}-\mathrm{NMR}: \delta$ 194.93, 178.62, 176.66, 147.06, 146.25, 133.97, 133.13, 132.73, 132.63, 131.08, 130.76, 128.74, 127.75, 127.53, 117.81, 116.94, 115.62, 43.85, 22.70. ESI-HRMS calcd. for $\mathrm{C}_{21} \mathrm{H}_{14} \mathrm{BrN}_{2} \mathrm{O}_{5} \mathrm{~S}_{3}{ }^{-}\left([\mathrm{M}-\mathrm{H}]^{-}\right)$: 548.9254; found: 548.9272.

2-(5-((6-Chloro-1-tosyl-1H-indol-3-yl)methylene)-4-oxo-2-thioxothiazolidin-3-yl)acetic acid (5d). Yellow solid; yield 57\%; m.p. $205^{\circ} \mathrm{C} .{ }^{1} \mathrm{H}-\mathrm{NMR}: \delta 2.35\left(\mathrm{~s}, 3 \mathrm{H}, \mathrm{CH}_{3}\right), 4.41\left(\mathrm{~s}, 2 \mathrm{H}, \mathrm{NCH}_{2}\right), 7.42-8.11(\mathrm{~m}, 9 \mathrm{H}, \mathrm{Ar}-\mathrm{H}$, $\mathrm{CH}=\mathrm{C}), 9.28$ (br.s, $1 \mathrm{H}, \mathrm{COOH}) .{ }^{13} \mathrm{C}-\mathrm{NMR}: \delta$ 192.52, 167.45, 166.62, 147.13, 134.63, 133.57, 131.44, 131.16, 128.36, 127.96, 127.79, 125.29, 124.48, 122.46, 121.63, 117.15, 113.30, 43.89, 22.73. ESI-HRMS calcd. for $\mathrm{C}_{21} \mathrm{H}_{14} \mathrm{ClN}_{2} \mathrm{O}_{5} \mathrm{~S}_{3}^{-}\left([\mathrm{M}-\mathrm{H}]^{-}\right)$: 504.9759; found: 504.9774 .

(R)-2-(5-((1-(Phenylsulfonyl)-1H-indol-3-yl)methylene)-4-oxo-2-thioxothiazolidin-3-yl)-4-methylpentanoic acid (6a). Yellow solid; yield 51\%; m.p. $271-276{ }^{\circ} \mathrm{C} .[\alpha]_{\mathrm{D}}^{20}:+35(\mathrm{c}=0.20, \mathrm{DMF}) .{ }^{1} \mathrm{H}-\mathrm{NMR}: \delta 0.89(\mathrm{~d}, 3 \mathrm{H}$, $\left.J=6.4 \mathrm{~Hz}, \mathrm{CHCH}_{3}\right), 0.94\left(\mathrm{~d}, 3 \mathrm{H}, J=6.4 \mathrm{~Hz}, \mathrm{CHCH}_{3}\right), 1.35-1.45\left(\mathrm{~m}, 1 \mathrm{H}, \underline{\mathrm{CHCH}_{3}}\right), 1.99-2.07(\mathrm{~m}, 1 \mathrm{H}$, $\left.\mathrm{CH}-\mathrm{H}_{\mathrm{a}}\right), 2.20-2.28\left(\mathrm{~m}, 1 \mathrm{H}, \mathrm{CH}-\mathrm{H}_{\mathrm{b}}\right), 5.61$ (br.s, $\left.1 \mathrm{H}, \overline{\mathrm{NCH}}\right), 7.38-8.20(\mathrm{~m}, 11 \mathrm{H}, \mathrm{Ar}-\mathrm{H}, \mathrm{CH}=\mathrm{C}), 13.31$ (br.s, $1 \mathrm{H}, \mathrm{COOH}) .{ }^{13} \mathrm{C}-\mathrm{NMR}: \delta$ 193.05, 169.83, 166.48, 136.66, 135.80, 134.29, 130.56, 129.03, 128.46, 127.72, $126.88,125.03,123.82,122.02,120.82,117.21,113.72,56.47,36.88,25.29,23.36,22.37$. ESI-HRMS calcd. for $\mathrm{C}_{24} \mathrm{H}_{21} \mathrm{~N}_{2} \mathrm{O}_{5} \mathrm{~S}_{3}{ }^{-}\left([\mathrm{M}-\mathrm{H}]^{-}\right)$: 513.0618; found: 513.0629.

(R)-2-(5-((6-Chloro-1-(phenylsulfonyl)-1H-indol-3-yl)methylene)-4-oxo-2-thioxothiazolidin-3-yl)-4-methylpentanoic acid (6b). Yellow solid; yield 53\%; m.p. $180-181{ }^{\circ} \mathrm{C} .[\alpha]_{\mathrm{D}}^{20}:+62.5\left(\mathrm{c}=0.20\right.$, DMF). ${ }^{1} \mathrm{H}-\mathrm{NMR}$ : $\delta 0.86\left(\mathrm{~d}, 3 \mathrm{H}, J=6.6 \mathrm{~Hz}, \mathrm{CHCH}_{3}\right), 0.91\left(\mathrm{~d}, 3 \mathrm{H}, J=6.6 \mathrm{~Hz}, \mathrm{CHCH}_{3}\right), 1.33-1.38\left(\mathrm{~m}, 1 \mathrm{H}, \underline{\mathrm{CHCH}_{3}}\right)$, 2.00-2.07 (m, 1H, CH-H $\left.\mathrm{a}_{\mathrm{a}}\right), 2.24-2.31\left(\mathrm{~m}, 1 \mathrm{H}, \mathrm{CH}-\mathrm{H}_{\mathrm{b}}\right), 5.50$ (br.s, 1 H, NCH) $7.20-8.22(\mathrm{~m}, 10 \mathrm{H}, \mathrm{Ar}-\mathrm{H}$, $\mathrm{CH}=\mathrm{C}), 9.30$ (br.s, $1 \mathrm{H}, \mathrm{COOH}) .{ }^{13} \mathrm{C}-\mathrm{NMR}: \delta$ 193.22, 170.01, 166.56, 136.51, 136.00, 134.63, 131.53, 130.70, $129.78,128.10,127.76,125.94,125.38,122.47,120.63,117.16,113.29,56.50,37.23,25.57,23.49,22.68$. ESI-HRMS calcd. for $\mathrm{C}_{24} \mathrm{H}_{20} \mathrm{ClN}_{2} \mathrm{O}_{5} \mathrm{~S}_{3}{ }^{-}\left([\mathrm{M}-\mathrm{H}]^{-}\right)$: 547.0228; found: 547.0244.

(R)-2-(5-((1-Tosyl-1H-indol-3-yl)methylene)-4-oxo-2-thioxothiazolidin-3-yl)-4-methylpentanoic acid (6c). Yellow solid; yield 66\%; m.p. $200{ }^{\circ} \mathrm{C}$. $[\alpha]_{\mathrm{D}}^{20}+45$ (c $=0.10$, DMF). ${ }^{1} \mathrm{H}-\mathrm{NMR}: \delta 0.88(\mathrm{~d}, 3 \mathrm{H}, J=6.4 \mathrm{~Hz}$, $\left.\mathrm{CHCH}_{3}\right), 0.93\left(\mathrm{~d}, 3 \mathrm{H}, J=6.5 \mathrm{~Hz}, \mathrm{CHCH}_{3}\right), 1.28-1.33\left(\mathrm{~m}, 1 \mathrm{H}, \underline{\mathrm{CHCH}_{3}}\right), 1.97-2.07\left(\mathrm{~m}, 1 \mathrm{H}, \mathrm{CH}-\mathrm{H}_{\mathrm{a}}\right)$,

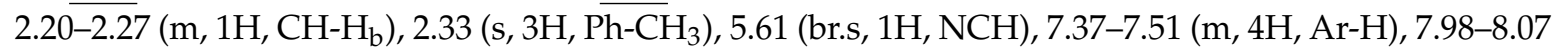
(m, 6H, Ar-H, CH=C), 13.04 (br.s, 1H, COOH). ${ }^{13} \mathrm{C}-\mathrm{NMR}: \delta 193.01,171.68,169.83,157.49,146.85,134.27$, $133.73,130.97,129.02,128.48,127.76,126.80,124.95,123.87,120.75,117.05,113.74,56.46,36.88,25.82$, 23.36, 22.37, 21.54. ESI-HRMS calcd. for $\mathrm{C}_{25} \mathrm{H}_{23} \mathrm{~N}_{2} \mathrm{O}_{5} \mathrm{~S}_{3}{ }^{-}\left([\mathrm{M}-\mathrm{H}]^{-}\right)$: 527.0775; found: 527.0790.

(R)-2-(5-((5-Bromo-1-tosyl-1H-indol-3-yl)methylene)-4-oxo-2-thioxothiazolidin-3-yl)-4-methylpentanoic acid (6d). Yellow solid; yield 61\%; m.p. 256-260 ${ }^{\circ} \mathrm{C}$. $[\alpha]_{\mathrm{D}}^{20}:+57.5$ (c = 0.08, DMF). ${ }^{1} \mathrm{H}-\mathrm{NMR}: \delta 0.89$ (d, 3H, $\left.J=6.3 \mathrm{~Hz}, \mathrm{CHCH}_{3}\right), 0.94\left(\mathrm{~d}, 3 \mathrm{H}, J=6.3 \mathrm{~Hz}, \mathrm{CHCH}_{3}\right), 1.45-1.53\left(\mathrm{~m}, 1 \mathrm{H}, \underline{\mathrm{CHCH}_{3}}\right), 1.99-2.08(\mathrm{~m}, 1 \mathrm{H}$,

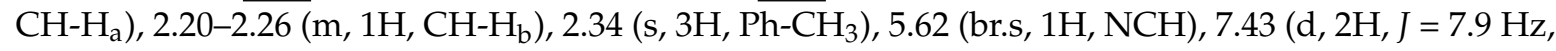
Ph-H), 7.61 (d, 1H, J = 8.7 Hz, Ph-H), 7.93-8.07 (m, 5H, Ar-H), 8.37 (s, 1H, CH=C), 13.23 (br.s, 1H, COOH). ${ }^{13}$ C-NMR: $\delta$ 193.01, 171.68, 169.83, 157.49, 146.85, 134.27, 133.73, 130.97, 129.02, 128.48, 127.76, $126.80,124.95,123.87,120.75,117.05,113.74,56.46,36.88,25.82,23.36,22.37,21.54$. ESI-HRMS calcd. for $\mathrm{C}_{25} \mathrm{H}_{22} \mathrm{BrN}_{2} \mathrm{O}_{5} \mathrm{~S}_{3}^{-}\left([\mathrm{M}-\mathrm{H}]^{-}\right)$: 604.9880; found: 604.9899 .

(R)-2-(5-((6-Chloro-1-tosyl-1H-indol-3-yl)methylene)-4-oxo-2-thioxothiazolidin-3-yl)-4-methylpentanoic acid (6e). Yellow solid; yield 63\%; m.p. $247-249{ }^{\circ} \mathrm{C} .[\alpha]_{\mathrm{D}}^{20}:+31.5\left(\mathrm{c}=0.20\right.$, DMF). ${ }^{1} \mathrm{H}-\mathrm{NMR}: \delta 0.88(\mathrm{~d}, 3 \mathrm{H}$, $\left.J=6.6 \mathrm{~Hz}, \mathrm{CHCH}_{3}\right), 0.94\left(\mathrm{~d}, 3 \mathrm{H}, J=6.6 \mathrm{~Hz}, \mathrm{CHCH}_{3}\right), 1.46-1.54\left(\mathrm{~m}, 1 \mathrm{H}, \underline{\mathrm{CHCH}_{3}}\right), 1.97-2.07(\mathrm{~m}, 1 \mathrm{H}$, $\left.\mathrm{CH}-\mathrm{H}_{\mathrm{a}}\right), 2.21-2.28\left(\mathrm{~m}, 1 \mathrm{H}, \mathrm{CH}-\mathrm{H}_{\mathrm{b}}\right), 2.34\left(\mathrm{~s}, 3 \mathrm{H},{\left.\mathrm{Ph}-\mathrm{CH}_{3}\right)}, 5.60\right.$ (br.s, $\left.1 \mathrm{H}, \mathrm{NCH}\right), 7.45(\mathrm{~d}, 3 \mathrm{H}, J=8.3 \mathrm{~Hz}$, Ar-H), 7.97-8.11 (m, 6H, Ar-H, CH=C), 13.04 (br.s, 1H, COOH). ${ }^{13} \mathrm{C}-\mathrm{NMR}: \delta$ 193.01, 169.80, 166.44, 147.16, 134.61, 133.54, 131.50, 131.13, 129.00, 127.88, 127.84, 125.34, 123.48, 122.51, 122.42, 116.93, 113.30, 56.46, 36.86, 25.28, 23.36, 22.36, 21.58. ESI-HRMS calcd. for $\mathrm{C}_{25} \mathrm{H}_{22} \mathrm{ClN}_{2} \mathrm{O}_{5} \mathrm{~S}_{3}{ }^{-}\left([\mathrm{M}-\mathrm{H}]^{-}\right): 561.0385$; found: 561.0406 . 
(S)-2-(4-Oxo-5-((1-(phenylsulfonyl)-1H-indol-3-yl)methylene)-2-thioxothiazolidin-3-yl)-3-phenylpropanoic acid (7a). Yellow solid; yield 58\%; m.p. $156-157{ }^{\circ} \mathrm{C} .[\alpha]_{\mathrm{D}}^{20}:-150$ (c=0.12, DMF). ${ }^{1} \mathrm{H}-\mathrm{NMR}: \delta 3.56(\mathrm{~d}, 2 \mathrm{H}$, $J=7.6 \mathrm{~Hz}, \mathrm{CHCH}_{2}$ ), 5.76 (br.s, 1H, NCH), 7.15-8.19 (m, 16H, Ar-H, CH=C), 8.85 (br.s, 1H, COOH). ${ }^{13}$ C-NMR: $\delta$ 192.67, 169.05, 166.55, 138.07, 136.66, 135.78, 134.23, 130.57, 129.32, 129.03, 128.73, 128.20, $127.74,126.93,126.85,124.99,122.82,122.17,120.77,117.11,113.70,44.05,22.70$. ESI-HRMS calcd. for $\mathrm{C}_{27} \mathrm{H}_{19} \mathrm{~N}_{2} \mathrm{O}_{5} \mathrm{~S}_{3}{ }^{-}\left([\mathrm{M}-\mathrm{H}]^{-}\right): 547.0462$; found: 547.0491 .

(S)-2-(5-((6-Chloro-1-(phenylsulfonyl)-1H-indol-3-yl)methylene)-4-oxo-2-thioxothiazolidin-3-yl)-3-phenylpropanoic acid $(7 \mathrm{~b})$. Yellow solid; yield 54\%; m.p. $220-224{ }^{\circ} \mathrm{C} .[\alpha]_{\mathrm{D}}^{20}:-144.5(\mathrm{c}=0.40, \mathrm{DMF}) .{ }^{1} \mathrm{H}-\mathrm{NMR}$ : $\delta 3.54$ (br.s, 2H, $\mathrm{CHCH}_{2}$ ), 5.91 (br.s, 1H, NCH), 7.19-8.24 (m, 15H, Ar-H, CH=C), 13.26 (br.s, 1H,

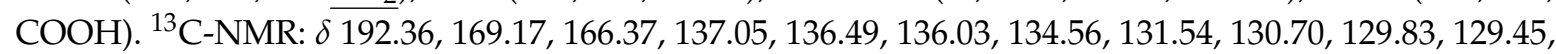
$128.93,128.78,128.47,127.85,127.20,125.39,123.23,122.52,116.87,113.25,44.85,25.77$. ESI-HRMS calcd. for $\mathrm{C}_{27} \mathrm{H}_{18} \mathrm{ClN}_{2} \mathrm{O}_{5} \mathrm{~S}_{3}{ }^{-}\left([\mathrm{M}-\mathrm{H}]^{-}\right)$: 581.0072; found: 581.0094 .

(S)-2-(5-((1-Tosyl-1H-indol-3-yl)methylene)-4-oxo-2-thioxothiazolidin-3-yl)-3-phenylpropanoic acid (7c). Yellow solid; m.p. $164-166{ }^{\circ} \mathrm{C}$, yield 55\%. $[\alpha]_{\mathrm{D}}^{20}:-174.25$ (c=0.40, DMF). ${ }^{1} \mathrm{H}-\mathrm{NMR}: \delta 2.32(\mathrm{~s}, 3 \mathrm{H}$, $\left.\mathrm{Ph}-\mathrm{CH}_{3}\right), 3.56\left(\mathrm{~d}, 2 \mathrm{H}, \mathrm{J}=7.0 \mathrm{~Hz}, \mathrm{CHCH}_{2}\right), 5.71$ (br.s, 1H, NCH), 7.14-7.50 (m, 9H, Ar-H), 7.90-8.05 (m, 6H, Ar-H, CH=C), 9.10 (br.s, 1H, C $\overline{\mathrm{OOH}}$ ). ${ }^{13} \mathrm{C}-\mathrm{NMR}: \delta$ 192.81, 169.00, 166.64, 146.83, 138.35, 134.21, $133.72,130.98,129.27,129.04,128.70,128.13,127.77,126.84,126.77,124.90,122.62,122.16,120.71$, $116.99,113.70,43.94,22.70,21.55$. ESI-HRMS calcd. for $\mathrm{C}_{28} \mathrm{H}_{21} \mathrm{~N}_{2} \mathrm{O}_{5} \mathrm{~S}_{3}{ }^{-}\left([\mathrm{M}-\mathrm{H}]^{-}\right)$: 561.0618 ; found: 561.06392.

(S)-2-(5-((5-Bromo-1-tosyl-1H-indol-3-yl)methylene)-4-oxo-2-thioxothiazolidin-3-yl)-3-phenylpropanoic acid (7d). Yellow solid; yield 59\%; m.p.217-221 ${ }^{\circ} \mathrm{C} .[\alpha]_{\mathrm{D}}^{20}:-216.25$ (c=0.08, DMF). ${ }^{1} \mathrm{H}-\mathrm{NMR}: \delta 2.34(\mathrm{~s}, 3 \mathrm{H}$, $\left.\mathrm{Ph}-\mathrm{CH}_{3}\right), 3.56\left(\mathrm{~d}, 2 \mathrm{H}, \mathrm{J}=6.8 \mathrm{~Hz}, \mathrm{CHCH}_{2}\right), 5.65$ (br.s, 1H, NCH), 7.15-7.63 (m, 9H, Ar-H), 7.86-8.05 (m, 4H, Ar-H), 8.35 (s, 1H, CH=C). ${ }^{13}$ C-NMR: $\delta$ 191.51, 173.04, 170.18, 148.15, 146.96, 142.46, 133.52, 133.24, 133.06, 131.08, 131.04, 129.33, 129.23, 128.68, 127.92, 127.80, 126.93, 126.82, 121.1, 117.9, 113.5, 44.13, 22.72, 22.17. ESI-HRMS calcd. for $\mathrm{C}_{28} \mathrm{H}_{20} \mathrm{BrN}_{2} \mathrm{O}_{5} \mathrm{~S}_{3}{ }^{-}\left([\mathrm{M}-\mathrm{H}]^{-}\right)$: 638.9723; found: 638.9745.

(R)-2-(4-Oxo-5-((1-(phenylsulfonyl)-1H-indol-3-yl)methylene)-2-thioxothiazolidin-3-yl)-3-phenylpropanoic acid (8a). Yellow solid; yield 65\%; m.p. $198-199^{\circ} \mathrm{C} .[\alpha]_{\mathrm{D}}^{20}:+255$ (c=0.02, DMF). ${ }^{1} \mathrm{H}-\mathrm{NMR}: \delta 3.57(\mathrm{~d}, 2 \mathrm{H}$, $J=6.8 \mathrm{~Hz}, \mathrm{CHCH}_{2}$ ), 5.63 (br.s, 1H, NCH), 7.13-8.17 (m, 16H, Ar-H, CH=C), 9.26 (br.s, 1H, COOH). ${ }^{13}$ C-NMR: $\delta$ 192.92, 169.15, 166.77, 139.01, 136.66, 135.76, 134.23, 130.56, 129.17, 129.07, 128.66, 127.93, $127.71,126.82,126.66,124.97,122.65,121.98,120.73,117.21,113.69,43.89,22.71$. ESI-HRMS calcd. for $\mathrm{C}_{27} \mathrm{H}_{19} \mathrm{~N}_{2} \mathrm{O}_{5} \mathrm{~S}_{3}{ }^{-}\left([\mathrm{M}-\mathrm{H}]^{-}\right)$: 547.0462; found: 547.0487.

(R)-2-(5-((6-Chloro-1-(phenylsulfonyl)-1H-indol-3-yl)methylene)-4-oxo-2-thioxothiazolidin-3-yl)-3-phenylpropanoic acid $(\mathbf{8 b})$. Yellow solid; yield 61\%; m.p. $264-265{ }^{\circ} \mathrm{C} .[\alpha]_{\mathrm{D}}^{20}:+170.36(\mathrm{c}=0.28, \mathrm{DMF}) .{ }^{1} \mathrm{H}-\mathrm{NMR}$ : $\delta 3.54\left(\mathrm{~d}, 2 \mathrm{H}, \mathrm{J}=6.9 \mathrm{CHCH}_{2}\right), 5.91$ (br.s, 1H, NCH), 7.20-8.23 (m, 15H, Ar-H, CH=C), 12.77 (br.s, $1 \mathrm{H}$, $\mathrm{COOH}) .{ }^{13} \mathrm{C}-\mathrm{NMR}: \delta$ 192.35, 169.15, 166.35, 137.00, 136.48, 136.04, 134.57, 131.55, 130.71, 129.45, 128.96, $128.79,127.85,127.21,125.39,123.29,122.54,122.25,116.86,113.26,100.00,58.76,29.48$. ESI-HRMS calcd. for $\mathrm{C}_{27} \mathrm{H}_{18} \mathrm{ClN}_{2} \mathrm{O}_{5} \mathrm{~S}_{3}{ }^{-}\left([\mathrm{M}-\mathrm{H}]^{-}\right)$: 581.0072; found: 581.0090 .

(R)-2-(5-((1-Tosyl-1H-indol-3-yl)methylene)-4-oxo-2-thioxothiazolidin-3-yl)-3-phenylpropanoic acid (8c). Yellow solid; yield 57\%; m.p. $176-181{ }^{\circ} \mathrm{C} .[\alpha]_{\mathrm{D}}^{20}:+178.25$ (c =0.40, DMF). ${ }^{1} \mathrm{H}-\mathrm{NMR}: \delta 2.32(\mathrm{~s}, 3 \mathrm{H}$, $\left.\mathrm{Ph}-\mathrm{CH}_{3}\right), 3.57$ (d, 2H, J = 7.1 Hz, $\left.\mathrm{CHCH}_{2}\right), 5.71$ (br.s, 1H, NCH), 7.14-7.50 (m, 9H, Ar-H), 7.90-8.05 (m, 6H, Ar-H, CH=C), 9.16 (br.s, 1H, $\overline{\mathrm{COOH}}) .{ }^{13} \mathrm{C}-\mathrm{NMR}: \delta 191.80,169.17,166.65,146.82,138.37,134.21$, 133.73, 130.97, 129.26, 129.04, 128.69, 128.12, 127.76, 126.83, 126.76, 124.89, 122.58, 122.17, 120.70, 116.99, 113.70, 43.90, 22.70, 21.54. ESI-HRMS calcd. for $\mathrm{C}_{28} \mathrm{H}_{21} \mathrm{~N}_{2} \mathrm{O}_{5} \mathrm{~S}_{3}{ }^{-}\left([\mathrm{M}-\mathrm{H}]^{-}\right)$: 561.0618; found: 561.0643 .

(R)-2-(5-((5-Bromo-1-tosyl-1H-indol-3-yl)methylene)-4-oxo-2-thioxothiazolidin-3-yl)-3-phenylpropanoic acid (8d). Yellow solid; yield 62\%; m.p. $192-193{ }^{\circ} \mathrm{C} .[\alpha]_{\mathrm{D}}^{20}:+159.13(\mathrm{c}=0.80, \mathrm{DMF}) .{ }^{1} \mathrm{H}-\mathrm{NMR}: \delta 2.34(\mathrm{~s}, 3 \mathrm{H}$, Ph- $\left.\mathrm{CH}_{3}\right), 3.56\left(\mathrm{~d}, 2 \mathrm{H}, \mathrm{J}=6.5 \mathrm{~Hz}, \mathrm{CHCH}_{2}\right), 5.71$ (br.s, 1H, NCH), 7.13-7.20 (m, 5H, Ar-H), 7.43 (d, 2H, $J=8.3 \mathrm{~Hz}, \mathrm{Ar}-\mathrm{H}), 7.61\left(\mathrm{dd}, 1 \mathrm{H}, J_{1}=\overline{8.9 \mathrm{~Hz}} J_{2}=1.8 \mathrm{~Hz}, \mathrm{Ar}-\mathrm{H}\right), 7.91-8.06(\mathrm{~m}, 5 \mathrm{H}, \mathrm{Ar}-\mathrm{H}), 8.37$ (s, $1 \mathrm{H}$, 
$\mathrm{CH}=\mathrm{C}) .{ }^{13} \mathrm{C}-\mathrm{NMR}: \delta 192.71,169.11,166.51,147.05,138.38,138.27,133.52,133.05,131.04,129.39,129.27$, 129.01, 128.70, 127.80, 126.86, 123.67, 122.62, 122.51, 117.80, 116.53, 115.57, 43.94, 22.71, 21.56. ESI-HRMS calcd. for $\mathrm{C}_{28} \mathrm{H}_{20} \mathrm{BrN}_{2} \mathrm{O}_{5} \mathrm{~S}_{3}{ }^{-}\left([\mathrm{M}-\mathrm{H}]^{-}\right)$: 638.9723; found: 638.9740 .

(R)-2-(5-((6-Chloro-1-tosyl-1H-indol-3-yl)methylene)-4-oxo-2-thioxothiazolidin-3-yl)-3-phenylpropanoic acid (8e). Yellow solid; yield 55\%; m.p. $277-280{ }^{\circ} \mathrm{C}$. $[\alpha]_{\mathrm{D}}^{20}:+182.5$ (c=0.80, DMF). ${ }^{1} \mathrm{H}-\mathrm{NMR}$ : $\delta 2.34(\mathrm{~s}, 3 \mathrm{H}$, Ph- $\left.\mathrm{CH}_{3}\right), 3.56$ (d, 2H, J = 7.0 Hz, $\mathrm{CHCH}_{2}$ ), 5.84 (br.s, 1H, NCH), 7.18-7.45 (m, 8H, Ar-H), 7.92-8.10 (m, 6H, Ar-H). ${ }^{13} \mathrm{C}-\mathrm{NMR}: \delta$ 192.32, 169.23, 166.43, 147.14, 137.43, 134.54, 133.53, 131.46, 131.12, 129.38, $128.88,128.76,127.87,127.09,125.30,122.97,122.49,122.34,116.76,113.26,110.60,33.77,29.48,21.58$. ESI-HRMS calcd. for $\mathrm{C}_{28} \mathrm{H}_{20} \mathrm{ClN}_{2} \mathrm{O}_{5} \mathrm{~S}_{3}{ }^{-}\left([\mathrm{M}-\mathrm{H}]^{-}\right)$: 595.0228; found: 595.0245.

2-(4-Oxo-5-((1-(phenylsulfonyl)-1H-indol-3-yl)methylene)-2-thioxothiazolidin-3-yl)3-methylbutanoic acid (9). Yellow solid; yield 46\%; m.p. $255-257{ }^{\circ} \mathrm{C} .{ }^{1} \mathrm{H}-\mathrm{NMR}: \delta 0.78\left(\mathrm{~d}, 3 \mathrm{H}, J=5.8 \mathrm{~Hz}, \mathrm{CHCH}_{3}\right), 1.22(\mathrm{~d}, 3 \mathrm{H}$, $\left.J=4.6 \mathrm{~Hz}, \mathrm{CHCH}_{3}\right), 2.77\left(\mathrm{~d}, 1 \mathrm{H}, \mathrm{CH}\left(\mathrm{CH}_{3}\right)_{2}\right), 5.21(\mathrm{~d}, 1 \mathrm{H}, J=7.5 \mathrm{~Hz}, \mathrm{NCH}), 7.41-8.20(\mathrm{~m}, 11 \mathrm{H}, \mathrm{Ar}-\mathrm{H}$, $\mathrm{CH}=\mathrm{C}$ ), 13.15 (br.s, $1 \mathrm{H}, \mathrm{COOH}) .{ }^{13 \mathrm{C}-\mathrm{NMR}} \delta$ 193.02, 169.09, 166.53, 136.67, 135.81, 134.29, 130.57, 129.03, $128.57,127.76,126.90,125.04,124.16,121.71,120.83,117.17,113.73,62.69,27.65,22.15,19.41$. ESI-HRMS calcd. for $\mathrm{C}_{23} \mathrm{H}_{19} \mathrm{~N}_{2} \mathrm{O}_{5} \mathrm{~S}_{3}{ }^{-}\left([\mathrm{M}-\mathrm{H}]^{-}\right)$: 499.0462; found: 499.0479 .

(S)-2-(4-Oxo-5-((1-(phenylsulfonyl)-1H-indol-3-yl)methylene)-2-thioxothiazolidin-3-yl)-3-methylpentanoic acid (10). Yellow solid; yield $42 \%$; m.p. $256-260{ }^{\circ} \mathrm{C} .[\alpha]_{\mathrm{D}}^{20}:-32.5\left(\mathrm{c}=0.4, \mathrm{CHCl}_{3}\right) .{ }^{1} \mathrm{H}-\mathrm{NMR}: \delta 0.82(\mathrm{t}, 3 \mathrm{H}$, $\left.J=7.2 \mathrm{~Hz}, \mathrm{CH}_{2} \mathrm{CH}_{3}\right), 0.94-1.01\left(\mathrm{~m}, 1 \mathrm{H}, \mathrm{CH}-\mathrm{H}_{\mathrm{a}}\right), 1.18\left(\mathrm{~d}, 3 \mathrm{H}, J=6.5 \mathrm{~Hz}, \mathrm{CHCH}_{3}\right), 1.47-1.53(\mathrm{~m}, 1 \mathrm{H}$,

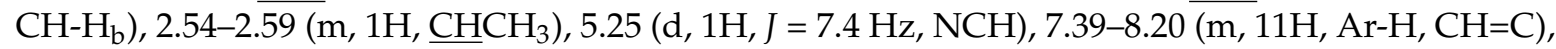
13.18 (br.s, $1 \mathrm{H}, \mathrm{COOH}) .{ }^{13} \mathrm{C}-\mathrm{NMR}: \delta$ 193.00, 169.09, 166.57, 136.65, 135.81, 134.27, 130.57, 129.02, 128.58, $127.75,126.89,125.03,124.19,121.66,120.84,117.17,113.71,62.15,33.53,25.37,18.05,11.34$. ESI-HRMS calcd. for $\mathrm{C}_{24} \mathrm{H}_{21} \mathrm{~N}_{2} \mathrm{O}_{5} \mathrm{~S}_{3}{ }^{-}\left([\mathrm{M}-\mathrm{H}]^{-}\right)$: 513.0629; found: 513.0618.

(S)-3-(4-Hydroxyphenyl)-2-(4-oxo-5-((1-(phenylsulfonyl)-1H-indol-3-yl)methylene)-2-thioxothiazolidin-3-yl) propanoic acid (11). Yellow solid; yield $47 \%$; m.p. $167-181{ }^{\circ} \mathrm{C} .[\alpha]_{\mathrm{D}}^{20}:-150(\mathrm{c}=0.2, \mathrm{DMF}) .{ }^{1} \mathrm{H}-\mathrm{NMR}$ : $\delta 3.46$ (br.s, $\left.2 \mathrm{H}, \mathrm{CHCH}_{2}\right), 5.69$ (br.s, $\left.1 \mathrm{H}, \mathrm{NCH}\right), 6.59$ (d, $\left.2 \mathrm{H}, J=8.1 \mathrm{~Hz}, \mathrm{Ar}-\mathrm{H}\right), 6.94$ (d, 2H, J = 8.1 Hz, Ar-H), 7.38-8.19 (m, 11H, Ar-H, CH=C), 9.13 (s, 1H, OH). ${ }^{13} \mathrm{C}-\mathrm{NMR}: \delta 192.67,170.93,166.57,156.25$, 136.66, 135.77, 134.22, 130.70, 130.56, 130.18, 129.05, 128.69, 128.16, 127.95, 127.75, 126.83, 124.98, 120.75, 117.10, 115.57, 113.69, 44.84, 25.78. ESI-HRMS calcd. for $\mathrm{C}_{27} \mathrm{H}_{19} \mathrm{~N}_{2} \mathrm{O}_{6} \mathrm{~S}_{3}{ }^{-}\left([\mathrm{M}-\mathrm{H}]^{-}\right)$: 563.0411; found: 563.0430 .

\subsection{Evaluation of Anti-Bacterial Activity In Vitro}

The anti-bacterial activity in vitro against S. aureus (CMCC(B) 26003 and CMCC 25923, S. pyogenes CMCC 32067, E. faecalis CMCC 29212, B. subtilis CMCC 63501; E. coli CMCC 25922 and CMCC 44568, P. aeruginosa CMCC 27853 and CMCC 10104, as well as two methicillin-resistant clinical isolates (S. aureus ATCC 43300 and ATCC 33591) was evaluated using a two-fold serial dilution technique [31], and the final concentrations of compounds obtained were in the range of $0.5-128 \mu \mathrm{g} / \mathrm{mL}$. Test bacteria were grown to mid-log phase in Mueller-Hinton broth (MHB) or Tryptone Soya Broth (TSB) and diluted 1000-fold in the same medium. The bacteria of $10^{5} \mathrm{CFU} / \mathrm{mL}$ were inoculated into MHB or TSB and dispensed at $0.2 \mathrm{~mL} /$ well in a 96-well microtiter plate. As positive controls, gatifloxacin, moxifloxacin, norfloxacin, oxacillin, and penicillin were used. Test compounds were prepared in DMSO, the final concentration of which did not exceed $0.05 \%$. The MIC was defined as the concentration of a test compound that completely inhibited bacteria growth during $24 \mathrm{~h}$ incubation at $37^{\circ} \mathrm{C}$. Bacteria growth was determined by measuring the absorption at $630 \mathrm{~nm}$ using a microtiter enzyme-linked immunosorbent assay (ELISA) reader. All experiments were carried out three times.

\subsection{Evaluation of Cytotoxicity In Vitro}

HEK 293 T cells were used to test the cytotoxicity of the new compounds. HEK 293T cells were grown in Dulbecco modified Eagle medium supplemented with fetal bovine serum (10\%), and 
antibiotics (penicillin-streptomycin mixture $(100 \mathrm{U} / \mathrm{mL}$ )). Cells at $80 \%$ to $90 \%$ confluence were split by trypsin (0.25\% in PBS; pH 7.4), and the medium was changed at $24 \mathrm{~h}$ intervals. The cells were cultured at $37^{\circ} \mathrm{C}$ in a $5 \% \mathrm{CO}_{2}$ incubator. The cells were grown to three passages, and approximately $1 \times 10^{4}$ cells were seeded into each well of a 96-well plate and allowed to incubate to allow attachment of the cells to the substrate. After $24 \mathrm{~h}$, the medium was replaced with DMEM supplemented with $10 \%$ FBS containing various concentrations $(4,8,16,32,64,128 \mu \mathrm{g} / \mathrm{mL})$ of test compounds and incubated for $48 \mathrm{~h}$. Each concentration set three wells in parallel. Then $20 \mu \mathrm{L}$ of CCK-8 solution was added to each well. After incubation for $3 \mathrm{~h}$, the optical density was measured at $450 \mathrm{~nm}$ using a microtiter ELISA reader. The $\mathrm{IC}_{50}$ values were defined as the concentrations inhibiting $50 \%$ of cell growth.

\section{Conclusions}

In summary, seven new series of $N$-arylsulfonylindoles 5-11 bearing rhodanine moieties were designed, synthesized, and evaluated for their antibacterial activities. In accordance to the results of antibacterial tests in vitro, some of the compounds showed good antibacterial activities against Staphylococcus aureus, including multidrug-resistant strains. Among them compounds 6a and 6c showed the most potent levels of activity $(\mathrm{MIC}=0.5 \mu \mathrm{g} / \mathrm{mL})$ against selected MRSA strains. These results illustrate that $N$-arylsulfonylindole analogs bearing rhodanine moieties are promising leads to develop novel antimicrobial agents against many infections caused by Gram-positive strains, especially Staphylococcus aureus, incluing MRSA. Future studies will focus on the mechanism of action of these compounds.

Acknowledgments: This work was supported by the National Science Foundation of China (No. 81560561) and Natural Science Foundation of Jiangxi Province, China (No. 20161BAB215207).

Author Contributions: Xian-Qing Deng designed experiments and conducted the spectrum analysis of target compounds; Wen-Hui Xu, Shao-Feng Xiong synthesized all the presented compounds; Ming-Xia Song, Song-Hui Li, Jiao-Yang Peng, and Ting-Ting Guo conducted the antimicrobial activity determination; Ming-Xia Song wrote the article, and made the discussion and conclusions.

Conflicts of Interest: The authors declare no conflict of interest.

\section{References}

1. Livermore, D.M. Has the era of untreatable infections arrived? J. Antimicrob. Chemother. 2009, 64 (Suppl. 1), i29-i36. [CrossRef] [PubMed]

2. Bi, Y.; Liu, X.X.; Zhang, H.Y.; Yang, X.; Liu, Z.Y.; Lu, J.; Lewis, P.J.; Wang, C.Z.; Xu, J.Y.; Meng, Q.G.; et al. Synthesis and Antibacterial Evaluation of Novel 3-Substituted Ocotillol-Type Derivatives as Leads. Molecules 2017, 22, 590. [CrossRef] [PubMed]

3. Carrel, M.; Perencevich, E.N.; David, M.Z. USA300 Methicillin-Resistant Staphylococcus aureus, United States, 2000-2013. Emerg. Infect. Dis. 2015, 21, 1973-1980. [CrossRef] [PubMed]

4. Hvistendahl, M. Public health. China takes aim at rampant antibiotic resistance. Science 2012, $336,795$. [CrossRef] [PubMed]

5. Yezli, S.; Li, H. Antibiotic resistance amongst healthcare-associated pathogens in China. Int. J. Antimicrob. Agents 2012, 40, 389-397. [CrossRef] [PubMed]

6. Azeredo da Silveira, S.; Perez, A. Liposomes as novel anti-infectives targeting bacterial virulence factors? Expert. Rev. Anti-Infect. Ther. 2015, 13, 531-533. [CrossRef] [PubMed]

7. Cascioferro, S.; Cusimano, M.G.; Schillaci, D. Antiadhesion agents against Gram-positive pathogens. Future Microbiol. 2014, 9, 1209-1220. [CrossRef] [PubMed]

8. Tay, S.B.; Yew, W.S. Development of quorum-based anti-virulence therapeutics targeting Gram negative bacterial pathogens. Int. J. Mol. Sci. 2013, 14, 16570-16599. [CrossRef] [PubMed]

9. Rademacher, J.; Welte, T. New antibiotics - standstill or progress. Med. Klin. Intensivmed. Notfmed. 2017, 112, 206-213. [CrossRef] [PubMed] 
10. Boucher, H.W.; Talbot, G.H.; Benjamin, D.K.; Bradley, J.; Guidos, R.J.; Jones, R.N.; Murray, B.E.; Bonomo, R.A.; Gilbert, D. Infectious Diseases Society of America. 10 x '20 Progress-development of new drugs active against gram-negative bacilli: an update from the Infectious Diseases Society of America. Clin. Infect. Dis. 2013, 56, 1685-1694. [CrossRef] [PubMed]

11. Bhale, P.S.; Chavan, H.V.; Dongare, S.B.; Shringare, S.N.; Mule, Y.B.; Nagane, S.S.; Bandgar, B.P. Synthesis of extended conjugated indolyl chalcones as potent anti-breast cancer, anti-inflammatory and antioxidant agents. Bioorg. Med. Chem. Lett. 2017, 27, 1502-1507. [CrossRef] [PubMed]

12. Diao, P.C.; Li, Q.; Hu, M.J.; Ma, Y.F.; You, W.W.; Hong, K.H.; Zhao, P.L. Synthesis and biological evaluation of novel indole-pyrimidine hybrids bearing morpholine and thiomorpholine moieties. Eur. J. Med. Chem. 2017, 13, 110-118. [CrossRef] [PubMed]

13. Manuel-Manresa, P.; Korrodi-Gregório, L.; Hernando, E.; Villanueva, A.; Martínez-García, D.; Rodilla, A.M.; Ramos, R.; Fardilha, M.; Moya, J.; Quesada, R.; et al. Novel indole-based tambjamine-analogues induce apoptotic lung cancer cell death through p38 mitogen-activated protein kinase activation. Mol. Cancer. Ther. 2017, in press. [CrossRef] [PubMed]

14. Barbosa, V.A.; Baréa, P.; Mazia, R.S.; Ueda-Nakamura, T.; Costa, W.F.; Foglio, M.A.; Goes Ruiz, A.L.; Carvalho, J.E.; Vendramini-Costa, D.B.; Nakamura, C.V.; et al. Synthesis and evaluation of novel hybrids $\beta$-carboline-4-thiazolidinones as potential antitumor and antiviral agents. Eur. J. Med. Chem. 2016, 124, 1093-1104. [CrossRef] [PubMed]

15. Kumar, V.P.; Renjitha, J.; Salfeena, C.F.; Ashitha, K.T.; Keri, R.S.; Varughese, S.; Sasidhar, B.S. Antibacterial and anti-tubercular evaluation of Dihydronaphthalenone-indole hybrid analogues. Chem. Biol. Drug. Des. 2017, in press. [CrossRef]

16. Patil, S.A.; Patil, S.A.; Patil, R. Medicinal applications of (benz)imidazole- and indole-based macrocycles. Chem. Biol. Drug. Des. 2017, 89, 639-649. [CrossRef] [PubMed]

17. Tan, M.C.; Wong, W.Y.; Ng, W.L.; Yeo, K.S.; Mohidin, T.B.; Lim, Y.Y.; Lafta, F.; Mohd Ali, H.; Ea, C.K. Identification of 5-Methoxy-2-(Diformylmethylidene)-3,3-Dimethylindole as an Anti-Influenza A Virus Agent. PLoS ONE 2017, 12, e0170352. [CrossRef] [PubMed]

18. Scuotto, M.; Abdelnabi, R.; Collarile, S.; Schiraldi, C.; Delang, L.; Massa, A.; Ferla, S.; Brancale, A.; Leyssen, P.; Neyts, J.; et al. Discovery of novel multi-target indole-based derivatives as potent and selective inhibitors of chikungunya virus replication. Bioorg. Med. Chem. 2017, 25, 327-337. [CrossRef] [PubMed]

19. Che, Z.; Tian, Y.; Hu, Z.; Chen, Y.; Liu, S.; Chen, G. Synthesis and in vitro anti-HIV-1 activity of a series of $\mathrm{N}$-arylsulfonyl-3-propionylindoles. Z. Naturforsch. C 2016, 71, 105-109. [CrossRef] [PubMed]

20. Silvestri, R.; De Martino, G.; La Regina, G.; Artico, M.; Massa, S.; Vargiu, L.; Mura, M.; Loi, A.G.; Marceddu, T.; La Colla, P. Novel indolyl aryl sulfones active against HIV-1 carrying NNRTI resistance mutations: Synthesis and SAR studies. J. Med. Chem. 2003, 46, 2482-2493. [CrossRef] [PubMed]

21. Vera, G.; Lagos, C.F.; Almendras, S.; Hebel, D.; Flores, F.; Valle-Corvalán, G.; Pessoa-Mahana, C.D.; Mella-Raipán, J.; Montecinos, R.; Recabarren-Gajardo, G. Extended N-Arylsulfonylindoles as 5-HT 6 Receptor Antagonists: Design, Synthesis \& amp; Biological Evaluation. Molecules 2016, 21, 1070.

22. Fan, L.L.; Liu, W.Q.; Xu, H.; Yang, L.M.; Lv, M.; Zheng, Y.T. Anti human immunodeficiency virus-1 (HIV-1) agents 3. synthesis and in vitro anti-HIV-1 activity of some $N$-arylsulfonylindoles. Chem. Pharm. Bull. (Tokyo) 2009, 57, 797-800. [CrossRef] [PubMed]

23. Xu, H.; Wang, Y.Y. Antifungal agents. Part 5: Synthesis and antifungal activities of aminoguanidine derivatives of $\mathrm{N}$-arylsulfonyl-3-acylindoles. Bioorg. Med. Chem. Lett. 2010, 20, 7274-7277. [CrossRef] [PubMed]

24. Song, M.X.; Deng, X.Q.; Li, Y.R.; Zheng, C.J.; Hong, L.; Piao, H.R. Synthesis And biological evaluation of (E)-1-(substituted)-3-phenylprop-2-en-1-ones bearing rhodanines as potent antimicrobial agents. J. Enzym. Inhib. Med. Chem. 2014, 29, 647-653. [CrossRef] [PubMed]

25. Song, M.X.; Zheng, C.J.; Deng, X.Q.; Wei, Z.Y.; Piao, H.R. The Synthesis and Anti-Bacterial Activities of N-carboxymethyl Rhodanines. Med. Chem. 2014, 4, 441-448.

26. Song, M.X.; Zheng, C.J.; Sun, L.P.; Wu, Y.; Hong, L.; Li, Y.J.; Liu, Y.; Wei, Z.Y.; Jin, M.J.; Piao, H.R. Synthesis and antibacterial evaluation of rhodanine-based 5-aryloxy pyrazoles against selected methicillin resistant and quinolone-resistant Staphylococcus aureus (MRSA and QRSA). Eur. J. Med. Chem. 2013, 60, 376-385. [CrossRef] [PubMed] 
27. Song, M.X.; Zheng, C.J.; Deng, X.Q.; Wang, Q.; Hou, S.P.; Liu, T.T.; Xing, X.L.; Piao, H.R. Synthesis and bioactivity evaluation of rhodanine derivatives as potential anti-bacterial agents. Eur. J. Med. Chem. 2012, 54, 403-412. [CrossRef] [PubMed]

28. Zheng, C.J.; Song, M.X.; Sun, L.P.; Wu, Y.; Hong, L.; Piao, H.R. Synthesis and biological evaluation of 5-aryloxypyrazole derivatives bearing a rhodanine-3-aromatic acid as potential antimicrobial agents. Bioorg. Med. Chem. Lett. 2012, 22, 7024-7028. [CrossRef] [PubMed]

29. Jin, X.; Zheng, C.J.; Song, M.X.; Wu, Y.; Sun, L.P.; Li, Y.J.; Yu, L.J.; Piao, H.R. Synthesis and antimicrobial evaluation of L-phenylalanine-derived C5-substituted rhodanine and chalcone derivatives containing thiobarbituric acid or 2-thioxo-4-thiazolidinone. Eur. J. Med. Chem. 2012, 56, 203-209. [CrossRef] [PubMed]

30. Chen, Z.H.; Zheng, C.J.; Sun, L.P.; Piao, H.R. Synthesis of new chalcone derivatives containing a rhodanine-3-acetic acid moiety with potential anti-bacterial activity. Eur. J. Med. Chem. 2010, 45, 5739-5743. [CrossRef] [PubMed]

31. Clinical and Laboratory Standards Institute. Methods for Dilution Antimicrobial Susceptibility Tests for Bacteria that Grow Aerobically, Approved Standard M7-A6; Clinical and Laboratory Standards Institute: Wayne, PA, USA, 2003.

Sample Availability: Samples of the compounds are available from the authors for a short period of time. 